A. Fiorenza and M. Krbec

Nagoya Math. J.

Vol. 158 (2000), 43-61

\title{
ON THE DOMAIN AND RANGE OF THE MAXIMAL OPERATOR
}

\author{
ALBERTO FIORENZA ${ }^{1}$ AND MIROSLAV KRBEC ${ }^{2}$
}

\begin{abstract}
We give a detailed survey, known and new results on the domain and the range of the maximal operator. In particular we employ the grand $L^{p}$ spaces and logarithmic Lebesgue spaces.
\end{abstract}

\section{$\S 1$. Introduction}

We shall systematically treat the problem of the domain and of the range of the maximal operator. Considering this problem has been motivated by questions how strong are assumptions about the maximal function, varying from the finiteness of the maximal function at a single point to $L_{r}$ integrability, where $0<r<1$, and questions about the range of the maximal operator under minimal necessary hypothesis about the space on which it acts, employing spaces obtained by various extrapolation procedures, in particular, $L^{p)}$ spaces (Iwaniec and Sbordone [IS]) and the logarithmic Lebesgue spaces (Edmunds and Triebel $[\mathrm{ET}]$ ).

We shall use standard notation $L^{p}$ for the Lebesgue spaces; we shall denote by $|E|$ the Lebesgue measure of a set $E \subset \mathbb{R}^{N}$ and by $B_{R}(x)$ the open ball centered at $x$ with radius $R$.

For $f \in L_{\text {loc }}^{1}\left(\mathbb{R}^{N}\right), N \geq 1$ we consider the (global, centered) maximal function

$$
M f(x)=\sup _{R>0} \frac{1}{\left|B_{R}(x)\right|} \int_{B_{R}(x)}|f(y)| d y, \quad x \in \mathbb{R}^{N},
$$

and we denote

$$
\mathbb{D}=\left\{f \in L_{\mathrm{loc}}^{1}\left(\mathbb{R}^{N}\right) ; M f \not \equiv \infty\right\} .
$$

Received July 16, 1998.

1991 Mathematics Subject Classification: Primary 42B25; Secondary 46E30.

${ }^{1}$ This work has been performed as a part of a National Research Project and partly supported by G.N.A.F.A.

${ }^{2}$ The research of the second author was partly supported by Grant No. 201/96/0431 of GA ČR.

We also gratefully appreciate a partial support of the NATO grant No. CRG970071. 
In view of Theorem 2.2 and the sublinearity of the maximal operator, $\mathbb{D}$ is a linear subspace of $L_{\text {loc }}^{1}\left(\mathbb{R}^{N}\right)$. We shall refer to it as the domain of the maximal operator.

Let us note that not every function in $L_{\text {loc }}^{1}\left(\mathbb{R}^{N}\right)$ is in $\mathbb{D}$ : it suffices to consider, for instance, the function norm in $\mathbb{R}^{N}$.

It is well known that using, more generally, balls containing $x$ or cubes (centered or off-centered with respect to $x$ ) with sides parallel to coordinate axes, or families of regular sets instead of balls in the definition of the maximal function yields an equivalent operator ([DS]).

For simplicity and without loss of generality we shall work only with non-negative functions so that we shall omit the absolute values everywhere.

In Section 2 we study the domain of the maximal operator, proving some necessary and sufficient conditions; after recalling that $L^{1}\left(\mathbb{R}^{N}\right) \subset$ $\mathbb{D}$, examples are given showing that $L_{\text {loc }}^{1}\left(\mathbb{R}^{N}\right) \cap \bigcup_{0<r<1} L^{r}\left(\mathbb{R}^{N}\right) \not \subset \mathbb{D} \not \subset$ $L^{1}\left(\mathbb{R}^{N}\right)+L^{\infty}\left(\mathbb{R}^{N}\right)$. In Section 3 we shall recall some known properties of $M f$ when $f \in \mathbb{D}$, and we shall fix our attention to the properties of $M f$ when $f \in L^{1}\left(\mathbb{R}^{N}\right)+L^{\infty}\left(\mathbb{R}^{N}\right)$. Among the consequences of our results, we get for instance that for every $0<r, s<1, \alpha>0$, we have

$$
f \in L^{r}\left(\mathbb{R}^{N}\right) \cap L_{\mathrm{loc}}^{1}\left(\mathbb{R}^{N}\right) \text { and } M f \in L^{s}(\{M f>\alpha\}) \Longrightarrow f \in L^{1}\left(\mathbb{R}^{N}\right) .
$$

In Section 4 we study the local maximal operator $M_{\Omega} f$ on open bounded sets $\Omega$ in $\mathbb{R}^{N}$. This operator is a powerful tool in many areas of Analysis, for instance, in the theory of quasi-conformal mappings ([BI]), when proving the regularity of solutions of P.D.E. or minimizers of functionals of the Calculus of Variations (for instance, see [FS1], [FS2]), or, recently, when dealing with homogenization problems without regularity in the coefficients $[\mathrm{CP}]$. We shall put in evidence the different properties with respect to the maximal operator in $\mathbb{R}^{N}$; we shall prove a characterization of the domain $\mathbb{D}_{\Omega}$ of the local maximal operator and we shall see some examples, for instance, functions in $\mathbb{D}_{\Omega} \backslash L_{\text {loc }}^{1}(\Omega)$. In our treatment the grand $L^{p}$ spaces (introduced in [IS]) and logarithmic Lebesgue spaces (see [ET]) will be involved.

\section{$\S 2$. The domain of the maximal operator}

In 1939 Wiener [Wie] found a subspace of $L_{\text {loc }}^{1}\left(\mathbb{R}^{N}\right)$ contained in $\mathbb{D}$; namely, he proved the following

Theorem 2.1. If $f \in L^{1}\left(\mathbb{R}^{N}\right)$, then $M f<\infty$ a.e. in $\mathbb{R}^{N}$. 
Plainly $L^{\infty}\left(\mathbb{R}^{N}\right) \subset \mathbb{D}$ so that by the sublinearity of the maximal operator $L^{1}\left(\mathbb{R}^{N}\right)+L^{\infty}\left(\mathbb{R}^{N}\right) \subset \mathbb{D}$. Therefore the Lebesgue $L^{p}\left(\mathbb{R}^{N}\right)(1 \leq p \leq \infty)$, Orlicz $L_{A}\left(\mathbb{R}^{N}\right)$ ( $A$ Young function) and Lorentz spaces are subsets of $\mathbb{D}$. We shall show that $\mathbb{D}$ is effectively larger than $L^{1}\left(\mathbb{R}^{N}\right)+L^{\infty}\left(\mathbb{R}^{N}\right)$, but it does not contain any space of the type $L_{\text {loc }}^{1}\left(\mathbb{R}^{N}\right) \cap\left(L^{r}\left(\mathbb{R}^{N}\right)+L^{\infty}\left(\mathbb{R}^{N}\right)\right)$ where $0<r<1$. We begin with the following characterization of $\mathbb{D}$.

THEOREM 2.2. Let $f \in L_{\text {loc }}^{1}\left(\mathbb{R}^{N}\right)$. Then the following statements are equivalent:

(i) there exists $x_{0} \in \mathbb{R}^{N}$ such that $M f\left(x_{0}\right)<\infty$;

(ii) there exists $x_{0} \in \mathbb{R}^{N}$ such that

$$
\limsup _{R \rightarrow \infty} \frac{1}{\left|B_{R}\left(x_{0}\right)\right|} \int_{B_{R}\left(x_{0}\right)} f(y) d y<\infty
$$

(iii) there exists $K>0$ such that

$$
\limsup _{R \rightarrow \infty} \frac{1}{\left|B_{R}\left(x_{0}\right)\right|} \int_{B_{R}\left(x_{0}\right)} f(y) d y=K<\infty
$$

for every $x_{0} \in \mathbb{R}^{N}$;

(iv) $M f(x)<\infty$ a.e. in $\mathbb{R}^{N}$;

(v) $f \in \mathbb{D}$.

Proof. The implications (iv) $\Rightarrow(v) \Rightarrow$ (i) are obvious. Also, if (i) holds, then the same $x_{0}$ satisfies (ii). We shall prove (ii) $\Rightarrow$ (iii) $\Rightarrow$ (iv).

Let (ii) hold and choose a sequence $\left\{\varrho_{n}\right\}_{n \geq 1}$ so that

$$
\lim _{n \rightarrow \infty} \frac{1}{\left|B_{\varrho_{n}}\left(x_{0}\right)\right|} \int_{B_{\varrho n}\left(x_{0}\right)} f(y) d y=\limsup _{R \rightarrow \infty} \frac{1}{\left|B_{R}\left(x_{0}\right)\right|} \int_{B_{R}\left(x_{0}\right)} f(y) d y
$$

and let $\bar{x}$ be any point in $\mathbb{R}^{N} \backslash\left\{x_{0}\right\}$. Since

$$
B_{\varrho_{n}+\left|\bar{x}-x_{0}\right|}(\bar{x}) \supset B_{\varrho_{n}}\left(x_{0}\right)
$$

we have $\left(\omega_{N}\right.$ denotes the measure of the unit ball in $\left.\mathbb{R}^{N}\right)$

$$
\frac{1}{\left|B_{\varrho_{n}+\left|\bar{x}-x_{0}\right|}(\bar{x})\right|} \int_{B_{\varrho_{n}+\left|\bar{x}-x_{0}\right|}(\bar{x})} f(y) d y
$$




$$
\begin{aligned}
& =\frac{1}{\omega_{N}\left(\varrho_{n}+\left|\bar{x}-x_{0}\right|\right)^{N}} \int_{B_{\varrho_{n}+\left|\bar{x}-x_{0}\right|}(\bar{x})} f(y) d y \\
& \geq \frac{1}{\omega_{N}\left(\varrho_{n}+\left|\bar{x}-x_{0}\right|\right)^{N}} \int_{B_{\varrho_{n}}\left(x_{0}\right)} f(y) d y \\
& =\left(\frac{\varrho_{n}}{\varrho_{n}+\left|\bar{x}-x_{0}\right|}\right)^{N} \frac{1}{\omega_{N} \varrho_{n}^{N}} \int_{B_{\varrho_{n}}\left(x_{0}\right)} f(y) d y .
\end{aligned}
$$

This yields

(2.1) $\limsup _{R \rightarrow \infty} \frac{1}{\left|B_{R}(\bar{x})\right|} \int_{B_{R}(\bar{x})} f(y) d y \geq \limsup _{R \rightarrow \infty} \frac{1}{\left|B_{R}\left(x_{0}\right)\right|} \int_{B_{R}\left(x_{0}\right)} f(y) d y$.

Replacing $\bar{x}$ by $x_{0}$ and vice versa in the above argument gives equality in (2.1), which proves (iii).

Let (iii) hold. Denote $A_{n}=\{n-1<|x|<n\}, n \in \mathbb{N}$. Then $f_{n}=f \chi_{A_{n}} \in$ $L^{1}$. By virtue of Theorem 2.1, $M f_{n}$ is finite a.e. in $\mathbb{R}^{N}$. Let $E_{n} \subset A_{n}$ be a set of zero measure such that $M f_{n}(x)<\infty$ for all $x \in A_{n} \backslash E_{n}$ and denote $E=\bigcup_{i=1}^{\infty} E_{i} \cup\left(\bigcup_{n \geq 0}\{|x|=n\}\right)$. We claim that $M f(x)<\infty$ for all $x \in \mathbb{R}^{N} \backslash E$. Let $x_{0} \in \mathbb{R}^{N} \backslash E$, then $x_{0} \in A_{n} \backslash E_{n}$ for some $n$. Let $r_{0}$ satisfy $B_{r_{0}}\left(x_{0}\right) \subset A_{n}$ and fix $R_{0}$ such that $\frac{1}{\left|B_{R}\left(x_{0}\right)\right|} \int_{B_{R}\left(x_{0}\right)} f(y) d y<2 K$ for all $R \geq R_{0}$. If $R \leq r_{0}$, then $\frac{1}{\left|B_{R}\left(x_{0}\right)\right|} \int_{B_{R}\left(x_{0}\right)} f(y) d y \leq M f_{n}\left(x_{0}\right)<\infty$; further, if $r_{0}<R<R_{0}$, then $\frac{1}{\left|B_{R}\left(x_{0}\right)\right|} \int_{B_{R}\left(x_{0}\right)} f(y) d y \leq \frac{1}{\left|B_{r_{0}}\left(x_{0}\right)\right|} \int_{B_{R_{0}}\left(x_{0}\right)} f(y) d y$, and, finally, if $R \geq R_{0}$, then $\frac{1}{\left|B_{R}\left(x_{0}\right)\right|} \int_{B_{R}\left(x_{0}\right)} f(y) d y<2 K$. Hence $M f\left(x_{0}\right)<\infty$ and (iv) is true.

Remark 2.3. Let $\Omega$ be an open set in $\mathbb{R}^{N}$. The John-Nirenberg space $\operatorname{BMO}(\Omega)$ is defined as the space of the measurable functions $f$ such that

$$
\|f\|_{\operatorname{BMO}(\Omega)}=\sup _{Q \subset \Omega} \frac{1}{|Q|} \int_{Q}\left|f(x)-\frac{1}{|Q|} \int_{Q} f(y) d y\right| d x<+\infty
$$

where the supremum is taken over all cubes $Q$ with sides parallel to the coordinate axes.

We remark that the implication (i) $\Rightarrow$ (iv) from the previous theorem has been shown to hold for $f \in \operatorname{BMO}\left(\mathbb{R}^{N}\right) \cap \mathbb{D}$ in the book by BennettSharpley (cf. [BS, pp. 399-400]), by using the inequality

$$
\frac{1}{|Q|} \int_{Q} M f(x) d x \leq c\|f\|_{\mathrm{BMO}\left(\mathbb{R}^{N}\right)}+\inf _{x \in Q} M f(x),
$$


true for every $Q$ cube in $\mathbb{R}^{N}, f \in \operatorname{BMO}\left(\mathbb{R}^{N}\right)$. Our proof here states this interesting property generally for $f \in L_{\text {loc }}^{1}\left(\mathbb{R}^{N}\right)$ and it is much simpler than that using (2.2). Notice that $\mathbb{D} \not \subset \mathrm{BMO}\left(\mathbb{R}^{N}\right)$ (see next Example 2.8). Also a rather sophisticated proof of (i) $\Rightarrow$ (iv) for $f \in L_{\text {loc }}^{1}\left(\mathbb{R}^{N}\right)$ was given by Wik [Wik].

Finally, we remark that it is also $\operatorname{BMO}\left(\mathbb{R}^{N}\right) \not \subset \mathbb{D}$; it suffices to consider $f=|\log | x||$ (cf. [BS, p. 400]).

We present two examples showing that if $0<r<1$, then we have $L_{\text {loc }}^{1}\left(\mathbb{R}^{N}\right) \cap L^{r}\left(\mathbb{R}^{N}\right) \not \subset \mathbb{D} \not \subset L^{1}\left(\mathbb{R}^{N}\right)+L^{\infty}\left(\mathbb{R}^{N}\right)$. Notice that the functions $f$ we are going to consider are supported on sets of finite measure, therefore, every level set $\{f(x)>\alpha\}, \alpha \geq 0$, is of finite measure.

ExAmple 2.4. Let $A_{n}=\{n-1<|x|<n\}, n \in \mathbb{N}$, and let $F_{n}$ be any measurable subset of $A_{n}$ such that $\left|F_{n}\right|=2^{-n}$. Put $f=\sum_{n=1}^{\infty} a_{n} \chi_{F_{n}}$ with $a_{n}=2^{n}$. Then $f \in L_{\text {loc }}^{1}\left(\mathbb{R}^{N}\right), f \notin L^{1}\left(\mathbb{R}^{N}\right)+L^{\infty}\left(\mathbb{R}^{N}\right)$. At the same time $f \in \mathbb{D}$, in fact,

$$
\frac{1}{\left|B_{\varrho}(0)\right|} \int_{B_{\varrho}(0)} f(y) d y \leq \frac{1}{\omega_{N} \varrho^{N}} \sum_{n=1}^{[\varrho+1]} a_{n}\left|F_{n}\right|, \quad \varrho>0
$$

where $[\cdot]$ denotes here the integer part and therefore we have

$$
\limsup _{\varrho \rightarrow \infty} \frac{1}{\left|B_{\varrho}(0)\right|} \int_{B_{\varrho}(0)} f(y) d y<\infty
$$

from which $f \in \mathbb{D}$ by Theorem 2.2. Notice that since $\int_{\mathbb{R}^{N}}(f(y))^{r} d y=$ $\sum_{n=1}^{\infty} a_{n}^{r}\left|F_{n}\right|<\infty$, we have $f \in L^{r}\left(\mathbb{R}^{N}\right)$ for all $0<r<1$.

ExAMPLE 2.5. Let us put $a_{n}=\left(2^{(r+1) / 2 r}\right)^{n}$ with some fixed $0<r<1$ in the previous example. Then $f \in L_{\text {loc }}^{1}\left(\mathbb{R}^{N}\right) \cap L^{r}\left(\mathbb{R}^{N}\right), f \notin \mathbb{D}$. Note that $f \notin L^{1}\left(\mathbb{R}^{N}\right)$.

Remark 2.6. Let $\Omega$ be an open set in $\mathbb{R}^{N}$. The space $\operatorname{BLO}(\Omega)$ is defined as the subspace of $\operatorname{BMO}(\Omega)$ of the functions $f$ such that

$$
\sup _{Q \subset \Omega}\left(\frac{1}{|Q|} \int_{Q} f(x) d x-\underset{Q}{\operatorname{essinf} f}\right)<+\infty .
$$

It is possible to prove that (cf. [BS, (8.38) p. 400]; see also [T, p. 204])

$$
M: \mathbb{D} \cap \operatorname{BMO}\left(\mathbb{R}^{N}\right) \longrightarrow \operatorname{BLO}\left(\mathbb{R}^{N}\right) \subset \operatorname{BMO}\left(\mathbb{R}^{N}\right)
$$


and that such operator is surjective, i.e.,

$$
\operatorname{BLO}\left(\mathbb{R}^{N}\right)=M\left(\mathbb{D} \cap \operatorname{BMO}\left(\mathbb{R}^{N}\right)\right)+L^{\infty}\left(\mathbb{R}^{N}\right)
$$

Remark 2.7. The Sobolev spaces are in $\mathbb{D}$, but also functions in $L_{\text {loc }}^{1}\left(\mathbb{R}^{N}\right)$ with weak derivatives in $L^{p}\left(\mathbb{R}^{N}\right), 1 \leq p<N$ are in $\mathbb{D}$ since they, up to a constant, belong to some Lebesgue or Orlicz space (cf. [Sc, p. 40], or [Ma, p. 424]).

ExAmple 2.8. Functions in $\mathbb{D}$ can be very bad indeed, for instance, the measure of every level set can be infinite, hence these functions cannot be rearranged (for general reference about rearrangements see for instance $[\mathrm{B}],[\mathrm{Mo}],[\mathrm{K}])$. Let us have a look at the following one-dimensional example: Put

$$
f(t)=\sum_{n=1}^{\infty} n \chi_{\left(n^{3}, n^{3}+1\right)}(t), \quad t \in \mathbb{R}^{1} .
$$

Clearly $f \in L_{\text {loc }}^{1}\left(\mathbb{R}^{1}\right)$ and

$$
\begin{aligned}
0 & \leq \lim _{t \rightarrow \infty} \int_{-t}^{t} f(y) d y \leq \lim _{n \rightarrow \infty} \frac{1}{2 n^{3}} \int_{-n^{3}-1}^{n^{3}+1} f(y) d y \\
& =\lim _{n \rightarrow \infty} \frac{n(n+1)}{4 n^{3}}=0
\end{aligned}
$$

therefore, $f \in \mathbb{D}$ by Theorem 2.2. Notice that the measure of every level set of $f$ is infinite, and, since $f \leq M f$, also the measure of every level set of $M f$ is infinite. Finally, notice that $f \notin \operatorname{BMO}\left(\mathbb{R}^{1}\right)$.

\section{$\S 3$. The range of the maximal operator}

We shall first survey known properties of the maximal operator. Let $f \in \mathbb{D}$. Then $M f$ is measurable lower semicontinuous function. By the Lebesgue Differentiation Theorem, $f \leq M f$, and if $N>2$ an equality can hold even without $f$ being a constant (this result is true for the spherical maximal operator; see $[\mathrm{F} 1])$. In terms of membership in various function spaces we recall that if $f \not \equiv 0$ a.e. in $\mathbb{R}^{N}$ then $M f \notin L^{r}\left(\mathbb{R}^{N}\right) \forall 0<r \leq 1$ and $\log (1+M f) \notin L^{1}\left(\mathbb{R}^{N}\right)$ (since there is $c>0$ such that $M f(x) \geq c /|x|^{N}$ for all $|x|>1$, cf., e.g., [BS, p. 117]). The behaviour of $M$ in Lebesgue spaces has been studied thoroughly: $M$ acts continuously from $L^{p}\left(\mathbb{R}^{N}\right)$ into $L^{p}\left(\mathbb{R}^{N}\right)$ if $1<p \leq \infty$ and from $L^{1}\left(\mathbb{R}^{N}\right)$ into the weak- $L^{1}\left(\mathbb{R}^{N}\right)$. Passing to 
finer scales of spaces, it is possible to find criteria for the boundedness of $M$ in Lorentz and Orlicz spaces, in particular then in Lorentz-Zygmund spaces. A curious phenomenon occurs, namely, a deterioration of the behaviour of $M$ near $L^{1}$, expressed in the classical form of the Zygmund inequality, a feature common to other classical operators. This is now well understood also in a more abstract setting as a general property of operators of weak type $(1,1)$ or its generalizations. From numerous references let us recall at least $[\mathrm{Gu}],[\mathrm{GCRF}],[\mathrm{T}],[\mathrm{BR}],[\mathrm{BS}],[\mathrm{KK}]$.

Now let us begin by studying the properties of $M f$. Starting only from the assumption $f \in \mathbb{D}$ it may happen that $M f \notin L_{\text {loc }}^{1}\left(\mathbb{R}^{N}\right)$; it suffices to consider the following well-known

EXAMPLE 3.1. Let us put $f(x)=\chi_{] 0,1 / 2[}(x) /\left(x \log ^{2} x\right), x \in \mathbb{R}^{1}$ a.e. (cf. [BS, p. 118], or [T, p. 77]). Then $f \in \mathbb{D}, M f \notin L_{\text {loc }}^{1}\left(\mathbb{R}^{N}\right)$. We observe that $M f \in L^{1}(\log L)^{-1}(] 0,1 / 2[)$.

Example 3.1 shows that also starting from $f \in L^{1}\left(\mathbb{R}^{N}\right)$ generally we have not $M f \in L_{\text {loc }}^{1}\left(\mathbb{R}^{N}\right)$. As we are going to see, in this case it is possible to find optimal spaces to which $M f$ belongs.

First of all, by virtue of the Kolmogorov inequality (cf., e.g., [Sa, Thm. 6.1, p. 190], or [S3, p. 43]),

$$
\left.\|M f\|_{L^{r}(A)}^{r} \leq \frac{c(N)|A|^{1-r}}{1-r}\|f\|_{L^{1}\left(\mathbb{R}^{N}\right)}^{r} \quad \forall f \in L^{1}\left(\mathbb{R}^{N}\right), r \in\right] 0,1[,
$$

true for every set $A \subset \mathbb{R}^{N},|A|<\infty$, the function $M f$ is in every $L^{r}(A)$ with $0<r<1$ provided $f \in L^{1}\left(\mathbb{R}^{N}\right)$, where $A$ is any set of $\mathbb{R}^{N}$ with finite measure. Furthermore, $M f$ belongs to any space on $A$ resulting from an extrapolation procedure based on (3.1), which preserves a finite (quasi-)norm on the right hand side. The extrapolation theory as developed up to now offers two reasonable candidates. The first is given by using an analogue of the approach considered for $p>1$ in [ET],

$$
\int_{0}^{\varepsilon_{0}} \varepsilon^{\sigma-1}\|M f\|_{L^{1-\varepsilon}(A)} d \varepsilon \leq c\left(N,|A|, \varepsilon_{0}, \sigma\right)\|f\|_{L^{1}\left(\mathbb{R}^{N}\right)}
$$

where $\varepsilon_{0} \in(0,1)$ is chosen arbitrarily, $\sigma>1$ is a parameter, or by using its discrete variant, the $\Sigma$-method due to Milman [Mi]. Going along the lines of the proof in $[\mathrm{ET}]$, where spaces $L^{p}(\log L)^{-\sigma}(A)$ with $1<p<\infty$ are considered, it is not difficult to prove that the quasinorm on the left hand 
side of (3.2) is equivalent to the quasinorm in the (generalized) Orlicz space $L^{1}(\log L)^{-\sigma}(A)$. Hence we have the following

Proposition 3.2. If $f \in L^{1}\left(\mathbb{R}^{N}\right)$, then $M f \in \bigcap_{\sigma>1} L^{1}(\log L)^{-\sigma}(A)$ for every $A \subset \mathbb{R}^{N}$ of finite measure.

This assertion is optimal in the scale of logarithmic Lebesgue spaces, as we are going to see from the following

EXAMPLE 3.3. Let us consider the function

$$
f(x)=\frac{1}{x|\log x| \log ^{2}|\log x|} \chi_{] 0, a[}(x), \quad x \in \mathbb{R}^{1} \backslash\{0,1, e\},
$$

where $a=\exp (-\exp (1))$. It is $f \in L^{1}\left(\mathbb{R}^{N}\right)$ because

$$
\int_{0}^{a} f(x) d x=\left[\frac{1}{\log |\log x|}\right]_{x \rightarrow 0}^{x=a}=\frac{1}{\log |\log a|} .
$$

On the other hand we have

$$
\left.M f\left(\frac{x}{2}\right) \geq \frac{1}{x} \int_{0}^{x} f(t) d t=\frac{1}{x \log |\log x|}, \quad x \in\right] 0, \frac{a}{2}[,
$$

and therefore, since $\log |\log x|>1 \forall x \in] 0, a / 2[$, we get

$$
\begin{aligned}
\frac{M f\left(\frac{x}{2}\right)}{\log \left(e+M f\left(\frac{x}{2}\right)\right)} & \geq \frac{\frac{1}{x \log |\log x|}}{\log \left(e+\frac{1}{x \log |\log x|}\right)} \geq \frac{1}{x \log |\log x| \log \left(e+\frac{1}{x}\right)} \\
& \geq \frac{1}{x \log |\log x| \log \frac{2}{x}} \geq \frac{1}{2 x|\log x| \log |\log x|}
\end{aligned}
$$

from which $M f \notin L^{1}(\log L)^{-1}(] 0, a[)$.

The second approach follows from another estimate consequence of (3.1), namely, a bound for the quasinorm of $M f$ in $L^{1)}(A)$, the grand $L^{1}$ space, which is a particular case of spaces introduced by Iwaniec and Sbordone [IS] and investigated in details, e.g., in Greco [Gr]:

$$
\|M f\|_{L^{1)}(A)} \leq c(N,|A|)\|f\|_{L^{1}\left(\mathbb{R}^{N}\right)}
$$


Let us recall that the quasinorm in $L^{1)}(A)$ is given by

$$
\|f\|_{L^{1)}(A)}=\sup _{0<\varepsilon<\varepsilon_{0}}\left(\varepsilon \frac{1}{|A|} \int_{A}|f(y)|^{1-\varepsilon} d y\right)^{1 /(1-\varepsilon)}
$$

where $\varepsilon_{0} \in(0,1)$; observe that only values of $\varepsilon$ near zero are relevant. Hence we have the following

Proposition 3.4. If $f \in L^{1}\left(\mathbb{R}^{N}\right)$, then $M f \in L^{1)}(A)$ for every $A \subset$ $\mathbb{R}^{N}$ of finite measure.

Let us remark that this approach is better in terms of inclusions of functions spaces, because it is

$$
L^{1}(\log L)^{-1}(A) \subset L^{1)}(A) \subset \bigcap_{\sigma>1} L^{1}(\log L)^{-\sigma}(A)
$$

for every $A$ of finite measure.

In Example 2.8 we showed that if $f \in \mathbb{D}$, then the measure of every level set of $M f$ can be infinite, considering a function $f$ having the same property. The next theorem shows among others that such phenomenon may occur even if the measure of every level set of $f$ is finite (namely, when $f \notin L^{1}\left(\mathbb{R}^{N}\right)+L^{\infty}\left(\mathbb{R}^{N}\right)$, as in the Example 2.4.

THEOREM 3.5. Let $f \in L_{\mathrm{loc}}^{1}\left(\mathbb{R}^{N}\right)$ and $\varphi:[0, \infty[\rightarrow[0, \infty[, \varphi$ strictly increasing, $\varphi(\infty)=\infty, \lim _{t \rightarrow \infty} \varphi(t) / t^{s}=0$ for some $0<s<1$. Then the following statements are equivalent:

(i) $f \in L^{1}\left(\mathbb{R}^{N}\right)+L^{\infty}\left(\mathbb{R}^{N}\right)$;

(ii) there is $\alpha>0$ such that $f \in L^{1}(\{f>\alpha\})$;

(iii) there is $\alpha>0$ such that $|\{M f>\alpha\}|<\infty$;

(iv) there is $\alpha>0$ and $0<r<1$ such that $M f \in L^{r}(\{M f>\alpha\})$;

(v) there is $\alpha>0$ such that $M f \in L^{r}(\{M f>\alpha\})$ for all $0<r<1$;

(vi) there is $0<r<1$ such that $M f \in L^{r}\left(\mathbb{R}^{N}\right)+L^{\infty}\left(\mathbb{R}^{N}\right)$;

(vii) $M f \in \bigcap_{0<r<1} L^{r}\left(\mathbb{R}^{N}\right)+L^{\infty}\left(\mathbb{R}^{N}\right)$;

(viii) $\varphi(M f) \in L^{1}\left(\mathbb{R}^{N}\right)+L^{\infty}\left(\mathbb{R}^{N}\right)$; 
(ix) there is $\alpha>0$ such that $|\{M f>\alpha\}|<\infty$ and

$$
M f \in L^{1)}(\{M f>\alpha\})+L^{\infty}\left(\mathbb{R}^{N}\right)
$$

(x) there is $\alpha>0$ such that $|\{M f>\alpha\}|<\infty$ and

$$
M f \in \bigcap_{\sigma>1} L^{1}(\log L)^{-\sigma}(\{M f>\alpha\})+L^{\infty}\left(\mathbb{R}^{N}\right) .
$$

Proof. We shall prove (i) $\Rightarrow$ (iii) $\Rightarrow$ (ii) $\Rightarrow$ (i) and (iii) $\Rightarrow$ (v) $\Rightarrow$ (viii) $\Rightarrow$ (iii) and (v) $\Rightarrow$ (vii) $\Rightarrow$ (vi) $\Rightarrow$ (iv) $\Rightarrow$ (iii) and (iii) $\Rightarrow$ (ix) $\Rightarrow$ (x) $\Rightarrow$ (vi).

Step 1 , (i) $\Rightarrow$ (iii): If $f \in L^{1}\left(\mathbb{R}^{N}\right)+L^{\infty}\left(\mathbb{R}^{N}\right)$, then there is $\alpha>0$ such that $f=f \chi_{\{f>\alpha\}}+f \chi_{\{f \leq \alpha\}} \equiv f_{1}+f_{2}$ with $f_{1} \in L^{1}\left(\mathbb{R}^{N}\right)$. We have $M f \leq M f_{1}+M f_{2} \leq M f_{1}+\alpha$ and thus $\{M f>\beta\} \subset\left\{M f_{1}+\alpha>\beta\right\}=$ $\left\{M f_{1}>\beta-\alpha\right\}$ for all $\beta \geq \alpha$.

Step 2, (iii) $\Rightarrow$ (ii): This is an immediate consequence of the reverse inequality of weak type $(1,1)$ of $M$, namely, there exist constants $c_{1}, c_{2}>0$ depending on $N$ such that (see [S3, p. 43], for instance):

$$
\left|\left\{x \in \mathbb{R}^{N}: M f(x)>c_{1} \alpha\right\}\right| \geq \frac{c_{2}}{\alpha} \int_{|f|>\alpha}|f| d x, \quad \forall \alpha>0 .
$$

Step 3, (ii) $\Rightarrow$ (i): It suffices to write $f=f \chi_{\{f>\alpha\}}+f \chi_{\{f \leq \alpha\}}$.

Step 4 , (iii) $\Rightarrow(\mathrm{v})$ : Fix $0<r<1$. By Step 2 there exists $\alpha>0$ such that $f \chi_{\{f>\alpha\}} \in L^{1}\left(\mathbb{R}^{N}\right)$, therefore

$$
\begin{aligned}
\int_{M f>\alpha}(M f)^{r} d x \leq \int_{M f>\alpha} & {\left[M\left(f \chi_{\{f>\alpha\}}\right)\right]^{r} d x } \\
& +\int_{M f>\alpha}\left[M\left(f \chi_{\{f \leq \alpha\}}\right)\right]^{r} d x<\infty .
\end{aligned}
$$

The first term on the right hand side is finite in view of the Kolmogorov inequality since $\{M f>\alpha\}$ has a finite measure.

Step $5,(\mathrm{v}) \Rightarrow$ (viii): Let $t_{s}(0<s<1)$ be such that $\varphi(t) \leq t^{s}, t \geq t_{s}$ and put $K=\max \left(t_{s}, \alpha\right)$. Then

$$
M f=(M f) \chi_{\{M f>K\}}+(M f) \chi_{\{M f \leq K\}} .
$$

Step 6, (viii) $\Rightarrow$ (iii): Let $\alpha>0$ be such that $\varphi(M f) \in L^{1}(\{M f>\alpha\})$. If $|\{M f>\alpha\}|=\infty$, then

$$
\int_{\{M f>\alpha\}} \varphi(M f) d x>\varphi(\alpha)|\{M f>\alpha\}|=\infty,
$$


which is a contradiction.

Step $7,(\mathrm{v}) \Rightarrow$ (vii): It suffices to write $M f=(M f)\left(\chi_{\{M f>\alpha\}}\right)+$ $(M f)\left(\chi_{\{M f \leq \alpha\}}\right)$.

Step 8 , (vii) $\Rightarrow($ vi): This is obvious.

Step 9, (vi) $\Rightarrow$ (iv): It suffices to apply (i) $\Rightarrow\left(\right.$ ii) to $(M f)^{r}$.

Step 10, (iv) $\Rightarrow$ (iii): It suffices to argue as in Step 6, replacing $\varphi(t)$ by $t^{r}$.

Step 11, (iii) $\Rightarrow$ (ix): Arguing as in Step 4 and using the quantitative form of the Kolmogorov inequality we get, for $0<r<1$,

$$
\begin{gathered}
\int_{M f>\alpha}(M f)^{r} d x \leq \int_{M f>\alpha}\left[M\left(f \chi_{\{f>\alpha\}}\right)\right]^{r} d x+\int_{M f>\alpha}\left[M\left(f \chi_{\{f \leq \alpha\}}\right)\right]^{r} d x \\
\leq \frac{c}{1-r}|\{M f>\alpha\}|^{1-r}\left\|f \chi_{\{f>\alpha\}}\right\|_{L^{1}(\{M f>\alpha\})}^{r}+\alpha^{r}|\{M f>\alpha\}|
\end{gathered}
$$

for some constant $c$ independent of $r$. Hence

$$
\begin{gathered}
\sup _{0<r<1-\varepsilon_{0}}\left(\frac{1-r}{|\{M f>\alpha\}|} \int_{M f>\alpha}(M f)^{r} d x\right)^{1 / r} \\
\leq c_{1}\left(\frac{\left\|f \chi_{\{f>\alpha\}}\right\|_{L^{1}(\{M f>\alpha\})}}{|\{M f>\alpha\}|}+1\right)
\end{gathered}
$$

for some $c_{1}$ independent of $r$, which yields $M f \in L^{1)}(\{M f>\alpha\})$. Hence $M f \in L^{1)}(\{M f>\alpha\})+L^{\infty}\left(\mathbb{R}^{N}\right)$.

Step $12,(\mathrm{ix}) \Rightarrow(\mathrm{x})$ : This follows by the known inclusion properties of $L^{1)}$ (see $\left.[\mathrm{Gr}]\right)$.

Step $13,(\mathrm{x}) \Rightarrow$ (vi): This follows by the known inclusion theorems of Orlicz spaces.

Remark 3.6. The condition (ii) in Theorem 3.5 says that $f$ is integrable over a special set of a finite measure. Examples 2.4 and 2.5 show that this cannot be replaced by integrability of $f$ over any set of finite measure. Furthermore, the condition (iii) in Theorem 3.5 implies that all level sets of the maximal functions in the examples recalled are of infinite measure.

Remark 3.7. Notice that assuming $f \in L^{1-\varepsilon}\left(\mathbb{R}^{N}\right) \cap L_{\mathrm{loc}}^{1}\left(\mathbb{R}^{N}\right)$ for some $\varepsilon \in(0,1)$ and $M f \in L^{r}(\{M f>\alpha\})$ for some $0<r<1$ and $\alpha>0$, then in view of Theorem 3.5 we have $f \in L^{1}\left(\mathbb{R}^{N}\right)$.

Next theorem is a well-known variant of Stein's $L \log L$ theorem ([S1], [S2]) for the maximal function. We state it here for completeness. 
TheOrem 3.8. Let $f \in L_{\mathrm{loc}}^{1}\left(\mathbb{R}^{N}\right)$ and $\sigma \geq 1$. Then the following statements are equivalent:

(i) $f[\log (1+f)]^{\sigma} \in L^{1}\left(\mathbb{R}^{N}\right)+L^{\infty}\left(\mathbb{R}^{N}\right)$;

(ii) there is $\alpha>0$ such that $f[\log (1+f)]^{\sigma} \in L^{1}(\{f>\alpha\})$;

(iii) there is $\alpha>0$ such that $M f \in L^{1}(\log L)^{\sigma-1}(\{f>\alpha\})$;

(iv) $M f \in L^{1}(\log L)^{\sigma-1}\left(\mathbb{R}^{N}\right)+L^{\infty}\left(\mathbb{R}^{N}\right)$.

What follows is a scheme of some of the sets of functions we have considered up to now, ordered by the strength of the conditions involved:

$$
\begin{aligned}
& f \text { measurable in } \mathbb{R}^{N} \\
& \longrightarrow \quad f \notin L_{\mathrm{loc}}^{1}\left(\mathbb{R}^{N}\right) \\
& f \in L_{\text {loc }}^{1}\left(\mathbb{R}^{N}\right) \\
& \downarrow \\
& f \in \mathbb{D} \\
& \downarrow \\
& \exists \alpha>0:|\{f>\alpha\}|<\infty \\
& \text { (Ex. 2.4) } \\
& |\{f>\alpha\}|=\infty \forall \alpha \geq 0 \\
& \overrightarrow{(E x .2 .8)} \\
& f \notin \mathbb{D} \\
& \overrightarrow{(E x .2 .5)} \\
& |\{M f>\alpha\}|=\infty \forall \alpha \geq 0 \\
& \exists \alpha>0:|\{M f>\alpha\}|<\infty \\
& \downarrow \\
& \exists \alpha>0: M f \in \frac{L^{1}}{\log L}(\{f>\alpha\}) \underset{(E x .3 .1)}{\longrightarrow} M f \notin L^{1}(\{f>\alpha\}) \forall \alpha \geq 0 \\
& \downarrow \\
& \exists \alpha>0: M f \in L^{1}(\{f>\alpha\}) \\
& \underset{(E x .3 .3)}{\longrightarrow} M f \notin \frac{L^{1}}{\log L}(\{f>\alpha\}) \forall \alpha \geq 0
\end{aligned}
$$

\section{$\S 4$. The local maximal function on open bounded sets}

The symbol $\Omega$ will now stand for an open bounded subset of $\mathbb{R}^{N}$, functions in $\Omega$ will be assumed to be measurable and non-negative. 
The local maximal function of $f$ is defined by

$$
M_{\Omega} f(x)=\sup _{\substack{Q \ni x \\ Q \subset \Omega \\ Q \text { cube }}} \frac{1}{|Q|} \int_{Q} f(y) d y, \quad x \in \Omega,
$$

where edges of cubes $Q$ are parallel with coordinate axes. If $\Omega \neq \mathbb{R}^{N}$, then $M_{\Omega}$ preserves only some of the properties of $M$ as we are going to see.

We shall first consider the case $\Omega=Q_{0}$, cube in $\mathbb{R}^{N}$. Putting

$$
\bar{f}= \begin{cases}f & \text { in } Q_{0}, \\ 0 & \text { in } \mathbb{R}^{N} \backslash Q_{0},\end{cases}
$$

it is easy to prove that

$$
M_{Q_{0}} f=\left.\left(M_{\mathbb{R}^{N}} \bar{f}\right)\right|_{Q_{0}} .
$$

As a consequence of Theorem 2.2, we have

Proposition 4.1. It is $M_{Q_{0}} f<\infty$ a.e. in $Q_{0}$ if and only if $f \in$ $L^{1}\left(Q_{0}\right)$

After the considerations of Section 3, we have some information about the range of $M_{Q_{0}}$ : if $f \in L^{1}\left(Q_{0}\right)$, then we have $M f \in L^{1)}\left(Q_{0}\right)$ and also that $M f \in \bigcap_{\sigma>1} L^{1}(\log L)^{-\sigma}\left(Q_{0}\right)$; in particular, $M f \in \bigcap_{0<r<1} L^{r}\left(Q_{0}\right)$. Also, Example 3.1 shows that $M f$ need not be locally integrable in $Q_{0}$.

Let us recall some known results about the range of $M_{Q_{0}}$ when $f$ is "better" than $L^{1}\left(Q_{0}\right)$ : by the Stein's $L \log L$ result [S1], $f \in L \log L\left(Q_{0}\right)$ iff $M_{Q_{0}} f \in L^{1}\left(Q_{0}\right)$ and $f$ belongs to the Orlicz space $L_{A}\left(Q_{0}\right)$, where $\inf _{t>0} t A^{\prime}(t) / A(t)>1$, iff $M_{Q_{0}}$ belongs to the same space. For other imbedding properties of the maximal operator see [T], [LZ], [GIM].

We shall turn our attention to the local maximal function with respect to a general domain $\Omega$. In this case $M_{\Omega}$ is different from $\left.\left(M_{\mathbb{R}^{N}} \bar{f}\right)\right|_{\Omega}$. In general $M_{\Omega} f \leq\left.\left(M_{\mathbb{R}^{N}} \bar{f}\right)\right|_{\Omega}$ and these functions need not be equivalent. We shall give an example of this curious phenomenon.

EXAMPLE 4.2. Let $N=2$,

$$
\Omega=\{z=(x, y) ;|z-1|<1\},
$$

and let $f=1$ in $\Omega \cap\{y>2 / \sqrt{5}\}$ and 0 otherwise in $\Omega$. Then $\left.\left(M_{\Omega} f\right)\right|_{\Omega \cap\{y \leq 0\}}=0$ while $\left.\left(M_{\mathbb{R}^{N}} \bar{f}\right)\right|_{\Omega \cap\{y \leq 0\}}>0$. 
Hence it turns out that the assumption $\bar{f} \in \mathbb{D}$ is a sufficient condition to get $f \in \mathbb{D}_{\Omega}$. Next example shows that such assumption is too strong when looking for the functions in $\mathbb{D}_{\Omega}$. A necessary and sufficient condition for a function $f$ to be in $\mathbb{D}_{\Omega}$ will be given in Theorem 4.5.

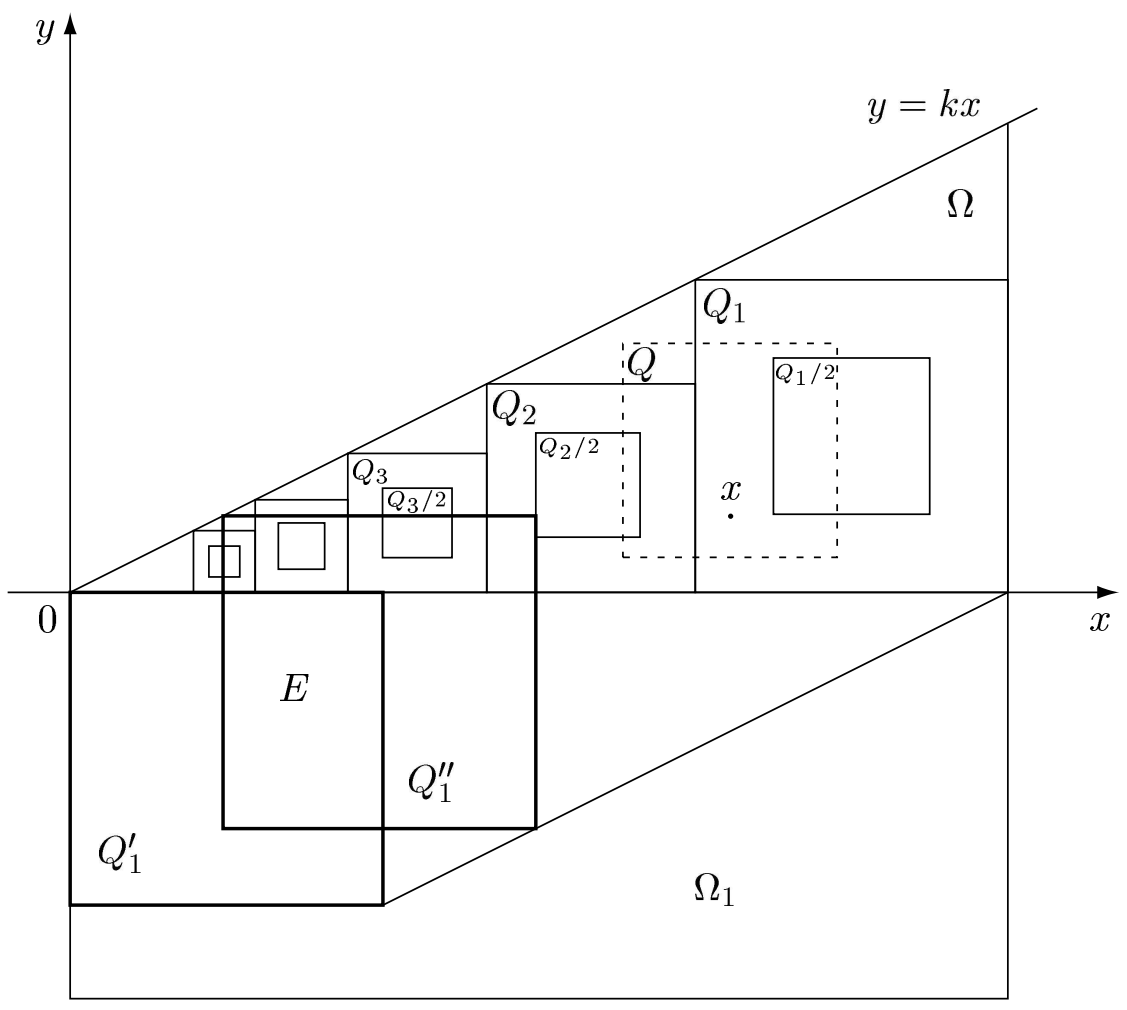

Figure 1.

EXAMPLE 4.3. Let us consider the situation illustrated by the upper part of Fig. 1, that is, let $N=2$ and consider a sequence of open cubes $Q_{1}, Q_{2}, \ldots$ such that their bottom sides lay on the positive axe $x$, the right vertical side of $Q_{k+1}$ is subset of the left vertical side of $Q_{k}$ and the left upper corners of all $Q_{k}$ lay on a line $y=k x$ with some $k>0$. Let $\Omega$ be the triangle domain whose boundary is contained in the positive axe $x$, the line $y=k x$, and the line containing the right vertical side of $Q_{1}$. Denote by $Q_{1} / 2, Q_{2} / 2, \ldots$, concentric cubes with sidelength equal to the half of the sides of $Q_{1}, Q_{2}, \ldots$ Let $\left(a_{i}\right)$, be any sequence of positive real numbers such 
that

$$
\sum_{i=1}^{\infty} a_{i}\left|Q_{i}\right|=\infty
$$

and put

$$
f=\sum_{i=1}^{\infty} a_{i} \chi_{Q_{i} / 2} .
$$

Then $f$ is supported in a compact set and $f \notin L^{1}(\Omega)$. If we fix $x \in \Omega$, then every cube $Q$ such that $Q \ni x, Q \subset \Omega$, intersect at most two of the cubes $Q_{i}$, thus $M_{\Omega}$ is finite a.e.

The preceding example shows that $f$ need not be integrable over every compact subset of its support in order to have $M_{\Omega} f<\infty$ a.e. in $\Omega$. Of course $f$ must be integrable over cubes contained in $\Omega$, this is, however, not sufficient for $M_{\Omega} f<\infty$ a.e. in $\Omega$. Indeed, $f$ can be integrable over cubes in $\Omega$, and still $M_{\Omega}$ need not be a.e. finite, as shown by the following

ExAMPLE 4.4. Let us consider $\Omega_{1}$ as $\Omega$ from Example 4.3 united with a rectangle pasted from below to $\Omega$, with the left vertical side on the axe $y$ and the upper horizontal side on the axe $x$ and put

$$
f=\sum_{i=1}^{\infty} \frac{i}{\left|Q_{i}\right|} \chi_{Q_{i} / 2}
$$

Let $Q_{1}^{\prime}$ be the translation of $Q_{1}$ having the left upper corner on the origin, and $Q_{1}^{\prime \prime} \neq Q_{1}^{\prime}$ be any fixed translation of $Q_{1}$, contained in $\Omega_{1}$, such that the left upper corner of $Q_{1}^{\prime \prime}$ stays on the line $y=k x$ and such that the set $E=Q_{1}^{\prime} \cap Q_{1}^{\prime \prime}$ has positive measure. Then for every $Q_{i}, i$ sufficiently large, there exists a translation of $Q_{1}$ containing $E \cup Q_{i}$, therefore, if $x \in E$, since

$$
M_{\Omega} f(x) \geq \frac{1}{\left|Q_{1}\right|} \int_{Q_{i}} f(y) d y=\frac{i}{4\left|Q_{1}\right|},
$$

we have $M_{\Omega} f=\infty$ in $E$. Hence $M_{\Omega} f$ is not finite a.e. while $f$ is integrable over every cube contained in $\Omega$.

Taking a closer look at the foregoing two examples we see that in both cases there exists a sequence of cubes such that the averages of $f$ blow up. In the former case the edges tend to zero and this is not necessarily in the latter example. It turns out that this property is in fact crucial for $\mathbb{D}_{\Omega}$; indeed, we have 
TheOREM 4.5. Let $f \in L^{1}(Q)$ for all $Q \subset \Omega$. Then the following statements are equivalent:

(i) $M_{\Omega} f<\infty$ a.e. in $\Omega$;

(ii) $\sup _{\substack{|Q|>\varepsilon \\ Q \text { cube } \\ Q \subset \Omega}} \frac{1}{|Q|} \int_{Q} f(y) d y<\infty$ for all $\varepsilon>0$.

Proof. Let (ii) hold and assume that $M_{\Omega}$ is not finite a.e. in $\Omega$. Then there exists a cube $C \subset \Omega$ such that $\left.\left(M_{\Omega} f\right)\right|_{C}$ is not finite a.e. Let

$$
C_{\varepsilon}=\{x \in C ; \operatorname{dist}(x, \partial C)>\varepsilon\}
$$

and choose $\varepsilon$ so that $\left|C \backslash C_{\varepsilon}\right|<\left|\left\{\left.\left(M_{\Omega} f\right)\right|_{C}=\infty\right\}\right|$. Since $f \in L^{1}\left(C_{\varepsilon}\right)$ we have $M_{C_{\varepsilon}} f<\infty$ a.e. in $C_{\varepsilon}$ and there exists $x \in C_{\varepsilon}$ such that $M_{\Omega} f(x)=\infty$ and $M_{C_{\varepsilon}} f(x)<\infty$. Consequently, one can find cubes $Q_{n} \subset \Omega$ such that $x \in \bigcap Q_{n},\left|Q_{n}\right|^{-1} \int_{Q_{n}} f(y) d y \rightarrow \infty$, and at the same time $Q_{n} \not \subset C_{\varepsilon}$ for every sufficiently large $n$, which yields $\left|Q_{n}\right|>\varepsilon$. This contradicts (ii), thus (i) holds.

Let us suppose that (i) is true and choose $\varepsilon_{0}$ such that

$$
\sup _{\substack{|Q|>\varepsilon_{0} \\ Q \text { cube } \\ Q \subset \Omega}} \frac{1}{Q} \int_{Q} f(x) d x=\infty
$$

further, let $Q_{n} \subset \Omega, n \in \mathbb{N}$, satisfy $\left|Q_{n}\right|^{-1} \int_{Q_{n}} f(x) d x \rightarrow \infty,\left|Q_{n}\right|>\varepsilon_{0}$. Denote by $x_{n}$ the centre of $Q_{n}$. Since $\Omega$ is bounded there exists a subsequence $\left(x_{n_{k}}\right)$ convergent to some $x \in \mathbb{R}^{N}$. But $x \in \Omega$ because $\left|Q_{n}\right|>\varepsilon_{0}$ for all $n$ and cubes $Q_{n_{k}}$ with sufficiently large $k$ contain the cube $Q\left(x, \varepsilon_{0}^{1 / N}\right)$ centered at $x$ and with sidelength $\varepsilon_{0}^{1 / N}$; hence $M_{\Omega} f(y)=\infty$ for all $y \in Q\left(x, \varepsilon_{0}^{1 / N}\right)$. This contradicts (i) and the proof is complete.

Remark 4.6. If $\Omega$ is a cube $Q_{0}$, then (ii) is equivalent to $f \in L^{1}\left(Q_{0}\right)$. Furthermore, Theorem 4.5 remains to be true for $\Omega=\mathbb{R}^{N}$ by virtue of Theorem 2.2.

Let us observe that the proof of Theorem 4.5 can be modified, admitting unbounded $\Omega$.

We turn our attention to the problem of the range of the local maximal function. If $f \in \mathbb{D}_{\Omega}$, then $M_{\Omega}$ trivially enjoys the standard properties, 
namely, it is lower semicontinuous and $f \leq M_{\Omega} f$ a.e. in $\Omega$. On the other hand, in contrast to the behaviour of $M_{\mathbb{R}^{N}}$ and $M_{Q_{0}}$, it is not generally true that $M_{\Omega} f \in L^{r}(\Omega)$. Let us consider, for instance, Example 4.3 with $a_{i}=i \exp \left(1 /\left|Q_{i}\right|^{2}\right)$. Then

$$
\int_{\Omega} f^{r} d x=\sum_{i=1}^{\infty} \frac{\left|Q_{i}\right|}{4} i^{r} \exp \left(\frac{r}{\left|Q_{i}\right|^{2}}\right)=\infty, \quad 0<r<1
$$

therefore $M_{\Omega} f \notin L^{r}(\Omega)$. This example indicates that the integrability properties of $M_{\Omega}$ depend heavily on the geometric properties of $\Omega$. In particular, if $\Omega$ is a cube $Q_{0}$, then $M_{Q_{0}} f \in L^{r}\left(Q_{0}\right)$ for all $0<r<1$.

Let us remark also that if $\Omega$ is a cube $Q_{0}$, in contrast to the behaviour of $M_{\mathbb{R}^{N}}, \operatorname{BMO}\left(Q_{0}\right) \subset \mathbb{D}_{Q_{0}}$ because $\operatorname{BMO}\left(Q_{0}\right) \subset L^{1}\left(Q_{0}\right)$, and, modulo bounded functions, $\operatorname{BLO}\left(Q_{0}\right)$ is exactly the range of $M_{Q_{0}}$ on $\operatorname{BMO}\left(Q_{0}\right)$ : in fact we have the following analogous of (2.3) (see [BS, p. 390])

$$
\operatorname{BLO}\left(Q_{0}\right)=M_{Q_{0}}\left(\operatorname{BMO}\left(Q_{0}\right)\right)+L^{\infty}\left(Q_{0}\right)
$$

In spite of this we have the following easy analogue of $(\mathrm{x})$ of Theorem 3.5:

Theorem 4.7. Let $f \in L^{1}(\Omega)$. Then $M_{\Omega} f \in L^{1)}(\Omega)$, therefore, $M_{\Omega} f \in$ $\bigcap_{\sigma>1} L^{1}(\log L)^{-\sigma}(\Omega)$

Proof. Let $Q_{0} \supset \Omega$ be a cube and put $\bar{f}(x)=f(x)$ for $x \in \Omega$ and $\bar{f}(x)=0$ in $\mathbb{R}^{N} \backslash \Omega$. The claims now follow since $M_{\Omega} f \leq M_{Q_{0}} \bar{f}=\left.\left(M_{\mathbb{R}^{N}} \bar{f}\right)\right|_{Q_{0}}$ and $f \in L^{1}\left(Q_{0}\right)$.

Remark 4.8. If $\Omega$ is a cube $Q_{0}$ and $M_{Q_{0}} f \in L^{1}(\log L)^{-1}\left(Q_{0}\right)$, then the $L^{1}$ norm of $f$ can be estimated as follows ([F2]):

$$
\int_{Q_{0}} f(x) d x \leq 2^{N+1} \int_{Q_{0}} \frac{M_{Q_{0}} f(x)}{\log \left(e+\frac{M_{Q_{0}} f(x)}{\left|M_{Q_{0}} f(x)\right|_{Q_{0}}}\right)} d x
$$

where $\left|M_{Q_{0}} f(x)\right|_{Q_{0}}=\left|Q_{0}\right|^{-1} \int_{Q_{0}} M_{Q_{0}} f(x) d x$. 


\section{REFERENCES}

[B] C. Bandle, Isoperimetric Inequalities and Applications, Monographs and Studies in Math. 7, Pitman, London, 1980.

[BI] B. Bojarski and T. Iwaniec, Analytical foundations of the theory of quasiconformal mappings in $\mathbb{R}^{N}$, Ann. Ac. Sc. Fen., 8 (1983), 257-324.

[BR] C. Bennett and K. Rudnick, On Lorentz-Zygmund spaces, Dissertationes Math. (Rozprawy Mat.), 175 (1980), 1-72.

[BS] C. Bennett and R. Sharpley, Interpolation of Operators, Academic Press, Boston, 1988.

[CP] L. A. Caffarelli and I. Peral, On $W^{1, p}$ estimates of elliptic equations in divergence form, Comm. Pure Appl. Math., 51 (1998), 1-21.

[DS] R. A. DeVore and R. C. Sharpley, Maximal functions measuring smoothness, American Mathematical Society, Memoirs of the AMS vol. 47, 293 (1984).

[ET] D. E. Edmunds and H. Triebel, Logarithmic Sobolev spaces and their applications to spectral theory, Proc. London Math. Soc. (3), 71 (1995), 333-371.

[F1] A. Fiorenza, A note on the spherical maximal function, Rend. Accad. Sci. Fis. Mat. Napoli, 65 (1987), 77-83.

[F2] A. Fiorenza, Some remarks on Stein's L $\log L$ result, Diff. and Int. Equations, 5 (6) (1992), 1355-1362.

[FS1] N. Fusco and C. Sbordone, Higher integrability from reverse Jensen inequalities with different supports, Partial Differential Equations and the Calculus of Variations: Essays in Honor of Ennio De Giorgi, Progress in Non-linear Differential Equations and their Applications, Birkhaüser Boston Inc. (1989), pp. 541-561.

[FS2] N. Fusco and C. Sbordone, Higher integrability of the gradient of minimizers of functionals with nonstandard growth conditions, Comm. Pure Appl. Math., 43 (1990), 673-683.

[GCRF] J. García-Cuerva and J. L. Rubio de Francia, Weighted Norm Inequalities and Related Topics, North Holland, Amsterdam, 1985.

[GIM] L. Greco, T. Iwaniec and G. Moscariello, Limits of the improved integrability of the volume forms, Indiana Univ. Math. J., 44 (1995), 305-339.

[Gr] L. Greco, A Remark on the Equality $\operatorname{det} D u=\operatorname{Det} D u$, Diff. and Int. Equations, 6 (1993), 1089-1100.

[Gu] M. de Guzmán, Differentiation of Integrals in $R^{n}$, Lecture Notes in Math. vol. 481, Springer-Verlag, Berlin-Heidelberg-New York, 1975.

[IS] T. Iwaniec and C. Sbordone, On the integrability of the Jacobian under minimal hypothesis, Arch. Rat. Mech. Anal., 119 (1992), 129-143.

[K] B. Kawohl, Rearrangements and Convexity of Level Sets in PDE, Lecture Notes in Math. vol. 1150, Springer Verlag, Berlin-New York, 1985.

[KK] V. Kokilashvili and M. Krbec, Weighted Inequalities in Lorentz and Orlicz Spaces, World Scientific, Singapore, 1991.

[LZ] C. Li and K. Zhang, Higher integrability of certain bilinear forms on Orlicz Spaces, Macquarie Math. Reports, Sydney (1994), pp. 94-157.

[Ma] V. G. Maz'ya, Sobolev Spaces, Springer-Verlag, Berlin, 1985. 
[Mi] M. Milman, Extrapolation and Optimal Decompositions, Springer-Verlag, Berlin, 1994.

[Mo] J. Mossino, Inégalités Isopérimétriques et Applications en Physique, Collection Travaux en Cours, Hermann, Paris (1984).

[Sa] C. Sadosky, Interpolation of Operators and Singular Integrals, M. Dekker Inc., New York, 1979.

[Sc] L. Schwartz, Théorie des Distributions. Tome II, Publications de l'Institut de Mathématique de l'Université de Strasbourg, vol X, Hermann, Paris, 1957.

[S1] E. Stein, Note on the class L $\log L$, Studia Math., 31 (1969), 305-310.

[S2] E. M. Stein, Singular Integrals and Differentiability Properties of Functions, Princeton Univ. Press, Princeton, New Jersey, 1970.

[S3] E. M. Stein, Harmonic Analysis: Real-Variable Methods, Orthogonality, and Oscillatory Integrals, Princeton Univ. Press, Princeton, New Jersey, 1993.

[T] A. Torchinsky, Real Variable Methods in Harmonic Analysis, Academic Press, San Diego, 1986.

[Wik] I. Wik, A comparison of the integrability of $f$ and $M f$ with that of $f^{\#}$, Preprint No. 2, University of Umeå (1983), pp. 1-30.

[Wie] N. Wiener, The ergodic theorem, Duke Math. J., 5 (1939), 1-18.

\author{
Alberto Fiorenza \\ Dipartimento di Costruzioni e Metodi Matematici in Architettura \\ via Monteoliveto, 3 \\ 80134, Napoli \\ Italy \\ fiorenza@cds.unina.it \\ Miroslav Krbec \\ Institute of Mathematics \\ Academy of Sciences of the Czech Republic \\ Žitná 25, 11567 Prague 1 \\ Czech Republic \\ krbecm@matsrv.math.cas.cz
}

